\title{
EL INSTITUTO PSICOLÓGICO ARGENTINO (1892). TEOSOFÍA, HIPNOSIS Y CHARLATANERÍA EN LOS ORÍGENES DE UNA INICIATIVA OLVIDADA
}

\author{
Mauro Vallejo \\ Consejo Nacional de Investigaciones Científicas y Técnicas (CONICET) \\ maurosvallejo@gmail.com \\ ORCID iD: https://orcid.org/0000-0002-1712-2181
}

Recibido: 22 junio 2016; Aceptado: 26 abril 2017.

Cómo citar este artículo/Citation: Vallejo (2018), “El Instituto Psicológico Argentino (1892). Teosofía, hipnosis y charlatanería en los orígenes de una iniciativa olvidada", Asclepio, 70 (1): p217. https://doi.org/10.3989/asclepio.2018.10

RESUMEN: En octubre de 1892 llegó a Buenos Aires un extraño personaje, que decía ser conde y poseer título médico. De inmediato comenzó a efectuar con la ayuda de su esposa demostraciones de hipnosis, de telepatía y de clarividencia. Ofreció además conferencias sobre esos asuntos. Muy pronto, a mediados de diciembre de ese año, fundó el Instituto Psicológico Argentino, tarea para la que contó con la colaboración de espiritistas y científicos locales. Todas esas actividades fueron informadas con detalle por los periódicos de la ciudad y por una de las revistas del espiritismo porteño. Al poco tiempo el Departamento de Higiene logró la clausura del Instituto, a pesar de lo cual Sgaluppi continuó con sus demostraciones y conferencias. El objetivo de este artículo es reconstruir en detalle esa historia, sobre todo con el auxilio de fuentes periódicas de la época. Nuestro cometido es iluminar un capítulo poco conocido de la historia del hipnotismo en Buenos Aires, poniendo de relieve dos aspectos: el valor que las disciplinas esotéricas tuvieron en la cultura científica de fines de siglo, y las dificultades que tuvieron las autoridades sanitarias para hacer valer sus regulaciones.

PALABRAS CLAVE: Hipnosis;Telepatía; Curanderismo; Espiritismo; Teosofía.

THE ARGENTINE PSYCHOLOGICAL INSTITUTE (INSTITUTO PSICOLÓGICO ARGENTINO) (1892). THEOSOPHY, HYPNOTISM AND QUACKERY IN THE ORIGINS OF A FORGOTTEN ENTERPRISE

In October 1892 a strange man arrived to Buenos Aires, who claimed to be a Viscount and to posses a medical degree. Immediately he began to perform with the help of his wife some demonstrations oh hypnotism, telepathy and clairvoyance. He also delivered lectures on these matters. Soon, in mid-December of that year, he founded the Argentine Psychological Institute (Instituto Psicológico Argentino), an enterprise for which he was assisted by local spiritualists and scientists. All these activities were reported in detail by local newspapers and by a spiritualist magazine. Soon the Health Office managed to close down the Institute, but nevertheless Sgaluppi continued with his performances and lectures. The aim of this paper is to explore those events, especially with the aid of primary sources. Our aim is to illuminate a tittle-known chapter in the history of Buenos Aires hypnotism, highlighting two aspects: the value that esoteric disciplines had in the fin-de-siècle scientific culture, and the difficulties experienced by health authorities to enforce their regulations.

KEY WORDS: Hypnotism; Telepathy; Quackery; Spiritualism; Theosophy.

Copyright: @ 2018 CSIC. Este es un artículo de acceso abierto distribuido bajo los términos de la licencia de uso y distribución Creative Commons Reconocimiento 4.0 Internacional (CC BY 4.0). 
A comienzos del siglo XX funcionó en Buenos Aires una temprana agrupación dedicada a difundir y desarrollar conocimientos y prácticas psicológicas. Nos referimos a la célebre Sociedad de Psicología de Buenos Aires, que perduró apenas unos cinco años (19081913). Nucleaba a distintos actores sociales (médicos, abogados, criminólogos, pedagogos) que por un motivo u otro mostraron un interés por alguna dimensión de lo que por entonces comenzaba a ser llamado psicología científica. La Sociedad contaba entre sus filas a importantes figuras del escenario intelectual, como por ejemplo José Ingenieros (1877-1925) o Francisco de Veyga (1866-1942), y publicó durante su corta vida unos Anales. En su seno reinó y se expandió el ideario positivista. En las últimas dos décadas muchos investigadores se han ocupado de ese capítulo de la historia de la ciencia en Argentina. Más puntualmente, entre los ensayos ligados a la historia de la psicología, una particular atención se ha prestado a la Sociedad nacida en 1908 (Ríos y Talak, 1999; Ostrovsky, 2008). Ahora bien, en la bibliografía especializada se postula, por regla general, que aquella agrupación fue la primera en su tipo en la ciudad de Buenos Aires y en Argentina. El objetivo de este artículo es ofrecer evidencias documentales sobre la existencia de una sociedad anterior, fundada en Buenos Aires en diciembre de 1892 y clausurada muy poco después (febrero de 1893). Se trató del Instituto Psicológico Argentino, que nació gracias a la iniciativa de un singular individuo proveniente de España, que se hacía llamar Alberto Martínez de Das, y que llegó al país ostentado credenciales de Conde y atribuyéndose título médico ${ }^{1}$. En rigor de verdad, ese sujeto, experto en hipnotismo y conocedor de las ciencias ocultas, carecía de títulos profesionales y jamás había pertenecido a la estirpe noble.

Tal y como intentaremos mostrar a continuación, la historia del Instituto compete tanto a la historia de la psicología argentina como a la historia de la medicina de ese país, y en no menor medida a la historia de las ciencias ocultas. En efecto, el fundador y director de esa prematura agrupación fue al mismo tiempo el primer propagandista de la teosofía en Buenos Aires. Fundó con la ayuda de su mujer la primera filial argentina de la teosofía, que de inmediato comenzó a difundir sus creencias por medio de la revista Luz. La escandalosa llegada a Buenos Aires de Martínez de Das (Ilamado en realidad Alberto Santini Sgaluppi) y su papel en la importación de la teosofía, han sido reconstruidos con mucho cuidado de manera reciente (Quereilhac, 2010). En esta oportunidad nos concentraremos en aquellas de sus iniciativas que todavía no han sido desentrañadas desde un punto de vista histórico: sus experiencias de hipnosis y su participación en la fundación del Instituto Psicológico Argentino.

A través del examen de los trabajos desplegados por aquel personaje en la capital argentina, espera- mos obtener una valiosa intelección de las dinámicas y los procesos de la cultura científica de fines de siglo. Por un lado, pretendemos sopesar con precisión el rol protagónico desempeñado por sujetos no diplomados en la difusión de saberes y vocabularios (ligados a la psicología o los automatismos nerviosos) que fueron inmediata y provechosamente reapropiados por médicos e intelectuales del medio local. Por otro lado, nuestro cometido es iluminar aspectos puntuales del mercado de la salud (o de la sanación) de ese período, caracterizado por la competencia constante entre actores sociales contrapuestos (médicos, curanderos, estafadores, hipnotizadores no diplomados, etc.). En efecto, el estudio de la presencia de Alberto Santini Sgaluppi (identificado en las fuentes de la época, por regla general, con su último apellido) habrá de aportarnos evidencias sustanciales sobre los obstáculos que impedían a los profesionales la imposición de las medidas represivas tendentes a refrenar el ejercicio ilegal de la medicina.

En las próximas páginas intentaremos explicar los motivos por los que un individuo como Sgaluppi pudo lograr una buena acogida de parte de la prensa de Buenos Aires, así como de ciertos profesionales de la ciudad, quienes lo acompañaron en la fundación y puesta en marcha del Instituto Psicológico Argentino. En el apartado que sigue analizaremos el pasado inmediato de aquel experto en hipnotismo. Antes de su llegada a la Argentina, Sgaluppi había logrado cierta notoriedad en España (sobre todo en Madrid) gracias a sus exhibiciones de hipnosis y telepatía. Entendemos que la reputación que muy pronto se ganaría en Buenos Aires se debió en parte a esas experiencias previas del otro lado del Atlántico. En efecto, apenas arribado a la capital argentina, Sgaluppi tuvo el cuidado de recuperar aquellos episodios en la península ibérica (sobre todo el interés que la Reina Isabel había manifestado por sus poderes), y para algunos porteños su nombre era ya conocido debido a aquellas peripecias de ultramar ${ }^{2}$. Por esas razones, y debido a que la recuperación de sus trabajos en España ha de servirnos para obtener un conocimiento bien claro de sus antecedentes y sus conocimientos, dirigiremos nuestra mirada a lo sucedido en aquellas latitudes.

\section{EL CONDE DE DAS Y LA HIPNOSIS EN ESPAÑA}

Por una razón de extensión, no podremos ofrecer en este artículo una reconstrucción detallada de los trabajos de Sgaluppi en España, ni podremos tampoco señalar de modo exhaustivo cómo se inscribe su nombre en la historia más general de la hipnosis en la Península Ibérica. Nos contentaremos con indicar los episodios más significativos.

Entre enero y julio de 1888 Sgaluppi realizó en Madrid cuantiosas demostraciones públicas, principal- 
mente en los salones particulares de algunos miembros de la aristocracia, y entre los asistentes se contaron políticos, médicos y personalidades de la cultura. Durante esos meses, importantes diarios de la ciudad brindaron crónicas sobre esas actividades, y dieron cuenta de la buena acogida que la familia real prestó a esas exhibiciones. Una de las primeras veladas se produjo el 8 de enero de 1888, en la casa de José López de Ayala. Según las crónicas, luego de una breve exposición oral, Das hipnotizó a una joven, en quien provocó una ostensible insensibilidad al dolor o a la electricidad, y a la que de inmediato colocó en estado de catalepsia o "rigidez cadavérica". Acto seguido sumió en hipnosis a una segunda joven, "le produjo primero el estado de éxtasis, la hizo bailar dormida, y por último, adivinar los papeles y contar los billetes de Banco que llevaba el Sr. D. Julián Zugasti [1836-1915] en un tarjetero guardado en el bolsillo de la levita" ${ }^{\text {. }}$

Con el correr de las semanas, Sgaluppi repetirá hasta la saciedad ese tipo de veladas. En todas ellas, además de disertar sobre la hipnosis y el magnetismo, exhibirá con la ayuda de "pacientes" o voluntarias los fenómenos más prodigiosos o enigmáticos: la adivinación del pensamiento, la doble vista, la transposición de sentidos, la anestesia absoluta. Martínez de Das tenía mucho cuidado en presentarse como un investigador, siempre recordaba que poseía título médico, y pretendía otorgar a sus actividades un cariz estrictamente científico. Tal vez por esos motivos, y quizá también debido a la novedad que significaba la hipnosis a los ojos de ciertos sectores de la sociedad española, el hipnotizador logró una visibilidad y un prestigio que muchos de sus colegas no tuvieron. El principal síntoma de esa reputación llegó muy pronto. Poco después de su arribo a la capital, fue invitado a realizar sus demostraciones ante la familia real. La velada fue desde todo punto de vista exitosa, y los diarios informaron en detalle sobre lo sucedido, y en las crónicas su puso especial énfasis en la participación activa de la reina en esas sesiones ${ }^{4}$.

En un momento en que la hipnosis comenzaba a ser mirada con recelo por algunos actores sociales (sobre todo por la Iglesia), y sobre todo en un momento en que se la emparentaba con tradiciones condenables (como el espiritismo y el charlatanismo), Sgaluppi parecía estar a resguardo de esas sospechas. Además de poseer título médico, era recibido por las mejores familias, y se ganaba la admiración de la ex reina regente. Evidencia de esto último fue que unos días más tarde Isabel le envío a Sgaluppi "el nombramiento de comendador de Isabel la Católica" a modo de agradecimiento ${ }^{5}$.

Sgaluppi no se dedicó solamente a divertir a los convidados a esas veladas en salones de la elite. Efectuó otras tareas que parecían reforzar su pertenencia a la disciplina científica, y que parecían encaminadas a enfatizar el carácter respetable de sus iniciativas. Por un lado, habría comenzado a publicar una revista semanal titulada La Hipnoterapia, que contenía textos propios y traducciones de trabajos de médicos franceses ${ }^{6}$. Por otro lado, dictó una conferencia en el Círculo Militar, siguiendo un programa elaborado con un vocabulario plagado de tecnicismos médicos: "Utilidades del hipnotismo como anestésico en las operaciones quirúrgicas. Anestesia e hiperestesia. Trasposiciones de los sentidos. Sonambulismo provocado y alucinaciones sonambúlicas. Sugestión mental y aparente. Excitaciones del sistema nervio-muscular. Atracción y repulsión. Encanto y éxtasis. Contracciones musculares y catalepsia" ${ }^{\prime \prime}$. Al igual que su labor en los salones reales, esa disertación de comienzos de febrero recibió también una fuerte atención de parte de la prensa ${ }^{8}$.

Trascurrido el primer semestre de 1888 el nombre de Das desaparece de las crónicas madrileñas. Es de suponer que sus experiencias habían perdido el brillo de la novedad. Por otro lado, por esas fechas se produjo una fuerte campaña en contra de la hipnosis de parte de la Iglesia, tal y como veremos a continuación. Por último, en ese tiempo, según algunas evidencias, Das realizó largos viajes por ciudades más pequeñas, con el objeto de realizar sus demostraciones. Recién en diciembre de 1890 nuestro personaje vuelve a aparecer en los periódicos, pero esta vez de una forma bien distinta. Por ese entonces Das es encarcelado, y junto con las noticias de su escandalosa detención se divulgan informaciones sobre su verdadera identidad $^{9}$. Según aquellas crónicas, los hechos que lo condujeron a la cárcel fueron los siguientes. Luego de haber establecido una academia de hipnotismo en la calle de Alcalá en Madrid, Das decidió mudarse al barrio del Pacífico y abrir allí un lujoso consultorio. Contrajo deudas para acondicionar el lugar, fue incapaz de pagarlas y sus acreedores acudieron a la justicia. Junto con informar de su reclusión, los diarios comunicaron que había quedado claro asimismo que el recluso no era Conde ni médico ${ }^{10}$.

Luego de ese escándalo, Sgaluppi se acercó a la filial española de la teosofía y en septiembre de 1892 una revista teosófica de Barcelona comunicó que en julio de ese año Das había sido expulsado del "Grupo Barcelonés" de la Sociedad debido a que había cometido "hechos graves", presuntamente de estafa"1.

No ha de sorprendernos que su nombre no haya aparecido en la historiografía hasta hace muy poco; falta, por ejemplo, en las documentadas reconstrucciones del desarrollo del hipnotismo publicadas hace más de una década (Diéguez Gómez, 2003; González de Pablo, 2003) ${ }^{12}$. En efecto, Das fue uno de los tantos magnetizadores e hipnotizadores que en la segunda 
mitad de la década de 1880 efectuó demostraciones públicas en ciudades ibéricas. Más allá de las crónicas periodísticas que aquí hemos recogido, poco rastro ha quedado de su utilización de la hipnosis. Dicho en otros términos, en comparación con otros actores sociales (médicos, abogados, miembros de la iglesia) que en esos mismos años escribieron abundantemente sobre hipnosis, la participación de Das en la difusión del sonambulismo artificial fue aparentemente minúscula. Los años en que nuestro personaje se mostró más hacendoso (1887-1889) son precisamente los años en que, debido mayormente a la llegada de traducciones de la obra de Bernheim, gran cantidad de obras sobre hipnosis se imprimieron en España, como La sugestión y sus aplicaciones a la terapéutica (1887) de Sánchez Calvo o La hipnología en nuestros días de Sánchez Freire (1888) (Diéguez, 2003, 219). Ahora bien, es posible ensayar un abordaje alternativo, y medir la significación del Conde de Das desde un ángulo distinto. Tal significación contempla dos aspectos complementarios: primero, Das fue, entre todos los hipnotizadores de teatro, el que mayor visibilidad adquirió en el momento en que esas experiencias batallaban por ganarse un lugar legítimo en la agenda científica española; segundo, la popularidad de sus sesiones fue lo que propició respuestas furibundas de parte de la Iglesia, respuestas encarnadas en textos que luego serían muy citados.

Respecto del primer punto, muchas de las crónicas de la época coinciden en atribuir a Das la popularización del hipnotismo entre el público madrileño ${ }^{13}$. Algunas fuentes fueron un poco más lejos, y afirmaron que gracias a las labores de Sgaluppi determinados científicos del momento se aproximaron a un campo de fenómenos que hasta entonces tenía una vida muy marginal ${ }^{14}$. En segundo lugar, las fuentes consultadas nos inducen a colegir que fueron los espectáculos de Das los que desencadenaron numerosos e influyentes textos contrarios a la hipnosis redactados por representantes de la Iglesia. Esa conexión es muy clara, por ejemplo, en las notas publicadas por Eugenio Fernández Hidalgo en La Unión Católica de fines de febrero de 1888 . Uno de esos textos, el del día 22, se tituló justamente "El Diablo en Madrid", y se refería a la responsabilidad que cabía atribuir a Das por la difusión del hipnotismo en la ciudad, caracterizado como un recurso peligroso y perjudicial para la salud, "la continuación de la magia antigua y de la pseudo-teurgia de los oráculos, la reproducción también de los sortilegios y brujerías de la Edad Media, y la prolongación, por último, del magneto-espiritismo". ${ }^{15}$ Por otro lado, no sería muy aventurado inferir que la carta pastoral redactada el 19 de marzo de 1888 por el obispo de Madrid Alcalá, fue en verdad una rápida respuesta de la curia a la popularidad que Sgaluppi acababa de ganarse entre los madrileños. En ese documento de 40 páginas, Ciríaco María Sancha y Hervás lanzaba de hecho una agria repulsa contra todo lo que tuviera olor a hipnotismo (Sancha y Hervás, 1888).

\section{UN HIPNOTIZADOR EN MUNDOS CONTRAPUESTOS}

En octubre de 1892, poco después de ser expulsado de la sociedad teosófica catalana, Sgaluppi desembarca en Buenos Aires en compañía de su esposa. La sociedad porteña le brindó una cálida acogida, y los diarios más prestigiosos de la ciudad siguieron de cerca sus primeros pasos. Según las crónicas que ahora revisaremos, nuestro personaje se presentó ante los habitantes de la capital argentina con sus clásicas credenciales: dijo llamarse Alberto Martínez de Das, ser Conde y poseer título médico. En una pequeña nota publicada por La Nación a los fines de darle la bienvenida al recién llegado, se informaba que era además "director de la Revista Científica de España"16.

Sin tiempo que perder, Sgaluppi realizó demostraciones de su arte. En efecto, el 31 de octubre llevó a cabo en el Hotel de Roma y junto a su esposa una primera sesión de hipnosis, telepatía, ocultismo y clarividencia, la cual fue seguida con mucho entusiasmo por el público letrado de la ciudad. Según el programa anunciado, en la parte teórica el conferenciante hablaría sobre algunos tópicos tradicionales, como las diferencias entre las escuelas de la Salpêtrière y de Nancy o las utilidades anestésicas del hipnotismo, pero también sobre problemáticas menos frecuentes en sesiones animadas por médicos, problemáticas aparentemente ligadas a las ciencias ocultas o el espiritismo: la fuerza néurica radiante de Barety, la visión a la distancia o "la psiquiatría y las ciencias de Oriente". ${ }^{17}$

El 1 de noviembre casi todos los diarios publicaron columnas detalladas y positivas sobre esa primera exhibición de Sgaluppi. Todos los cronistas atendieron al modo en que el conde se presentó ante los asistentes: mientras ostentaba en su pecho muchas condecoraciones y medallas, mostró al público folletos y libros presuntamente de su autoría. El Correo Español, por ejemplo, se refirió a la "notabilísima y brillante conferencia práctica sobre hipnotismo" brindada por el extranjero ${ }^{18}$. El Diario informó que entre los asistentes había abogados y médicos, y detalló los fenómenos exhibidos por la condesa colocada en estado de hipnosis: fue capaz de adivinar mentalmente y ejecutar órdenes que los invitados debían escribir en pequeños trozos de papel, jugó al dominó sin cometer errores a pesar de llevar sus ojos vendados por tres pañuelos, y otras maravillas. El Diario agregaba que Das tenía pensado radicarse en Buenos Aires y revalidar su título profesional ${ }^{19}$. La Prensa, por entonces el diario más venido en el país, distribuyó el relato más extenso y detallado sobre las demostraciones prácticas, en 
un artículo que no hacía otra cosa que festejar las capacidades y conocimientos del anfitrión ${ }^{20}$.

Sin embargo, no todos los periódicos miraron los fenómenos con ojos entusiastas. Fue el caso de La Nación, que lamentó que una demostración así fuera hecha en un salón lleno de curiosos, y no en un auditorio hospitalario o un aula de medicina ${ }^{21}$. Esa queja no era nueva, y tampoco eran muy nuevas las maravillas exhibidas por Sgaluppi. No era la primera vez que los porteños tenían ocasión de observar con sus propios ojos los milagros del hipnotismo, la clarividencia o la telepatía ${ }^{22}$. Tampoco era la primera vez en que algunas voces se alzaban para condenar la explotación teatral del hipnotismo. Desde finales de la década de 1880, diversos periódicos habían explicitado esa condena cada vez que un hipnotizador de teatro visitaba la ciudad. Más aún, en diciembre de 1890 el Departamento Nacional de Higiene había dictado una resolución que prohibía expresamente ese uso teatral del hipnotismo ${ }^{23}$.

Lo que sí era novedoso en el accionar de Sgaluppi era el modo en que él parecía habitar territorios divergentes. Sus faenas parecían combinar tradiciones que normalmente permanecían parcialmente distantes: medicina, espectáculo, ocultismo y espiritismo. Sería erróneo suponer que esas tradiciones nunca se mixturaban: los ilusionistas de teatro a veces usaban el vocabulario de la medicina (o afirmaban que su trabajo pertenecía al ámbito de la ciencia), o incluso había médicos que realizaban exhibiciones muy emparentadas a la vida teatral ${ }^{24}$. De todas maneras, habitualmente era claro y visible con qué tradición tal o cual hipnotizador se identificada. Alberto Sgaluppi vino a romper esa costumbre, y podemos conjeturar que una de las razones de su éxito (medido en la cantidad de labores que realizó) radicó en su habilidad para habitar simultánea o sucesivamente linderos contrapuestos ${ }^{25}$.

Ya en el anuncio de su primera conferencia el lenguaje del hipnotismo médico convivía con los términos de la teosofía o el ocultismo (por ejemplo, la fuerza radiante). Y con el correr de los días esa mezcla de registros se hará cada vez más patente. Más aún, el Instituto Psicológico Argentino nacerá en esa confluencia.

Unos días después de presentarse ante los porteños como médico, Sgaluppi publicó un primer escrito en la revista espiritista más importante de la ciudad, Constancia. El breve texto, titulado "Salve", llevaba la firma "Dr. A. M. de Das". Suerte de carta de presentación en sociedad, esas pocas columnas combinaban el anuncio de los objetivos de la tarea intelectual del autor, con una prédica moral en contra del egoísmo y a favor del bien común. Sgaluppi se definía allí como un trabajador de la ciencia y de la verdad, y enemi- go de la explotación teatral de ciertos conocimientos: "Es tiempo que los hombres de saber y buena fe la estudien [a la Psicología] en todos sus alcances y la defiendan como propiedad del ego individual, a la vez que cuiden meramente de que ésta no se enrede y manche en los laberintos de la especulación vulgar y de los espectáculos de fascinación o ilusión pseudohipnótica"26. Por otro lado, se otorgaba a la psicología un papel redentor, más próximo al terreno de la fe que al de la ciencia: "La luz se extenderá sobre la inmensidad, el destino del hombre no será un problema y el arte de conservar la salud a la pobre humanidad tendrá la sanción universal".

Podemos indicar una razón de peso por la cual la publicación espiritista acogió sin titubear el texto de Sgaluppi, y también se encargó de dar visibilidad a la labor del extranjero. El hecho de que el visitante poseyera título médico resultaba un fuerte atractivo para los kardecianos porteños, ante todo en ese momento en particular. En efecto, por esas semanas había recobrado actualidad el viejo tópico de la relación entre espiritismo y locura. Esa supuesta relación ya había sido esgrimida en la década anterior por importantes alienistas locales, como Lucio Meléndez y Wilfrido Rodríguez de la Torre (Rodríguez de la Torre, 1889) ${ }^{27}$. Pues bien, desde comienzos de octubre esas acusaciones habían vuelto a cobrar fuerza como consecuencia de un episodio que aquí no podremos reconstruir en detalle. El 5 de octubre de ese año una mujer atentó fallidamente contra la vida de Cosme Mariño (18471927), el líder de la sociedad espiritista Constancia. Tanto en las crónicas periodísticas que informaron sobre el hecho como en el informe médico-legal sobre la acusada, se intentó demostrar que el arrebato homicida había sido una consecuencia de la creencia en las ideas espiritistas. El propio Cosme Mariño se puso a la tarea de desmentir ese argumento, y durante varias semanas publicó, tanto en diarios locales como en la revista Constancia, textos que se ocupaban ya sea de señalar las falencias lógicas del informe de los médicos, ya de aportar evidencias que refutaban la pertenencia al espiritismo de la victimaria. No cabe dudar de que en esa coyuntura, el deseo del "médico" Martínez de Das de colaborar en las páginas de Constancia fue más que bienvenido por los responsables de la publicación.

Otro detalle contextual sirve para comprender de manera más cabal algunas de las conductas de Sgaluppi. El 20 de noviembre apareció en La Prensa una nota que informaba que hacía poco el "profesor de psicología" (y "comendador de la Real Orden de Isabel la Católica") se había acercado por iniciativa propia a la redacción del diario a los fines de aclarar que "nunca su propaganda con respecto a ciencias tan elevadas como el hipnotismo, la sugestión, espiritismo, etc., ha figurado en los carteles de un teatro" ${ }^{\prime 28}$. ¿Por qué mo- 
tivo nuestro personaje realizó tal aclaración, que en verdad venía a enfatizar una advertencia ya introducida en su texto del día 13 de noviembre? El propio artículo de La Prensa se encargaba de esclarecerlo. El gesto de Das se motivaba en la noticia que había circulado en días previos, según la cual el Departamento de Higiene prohibía al "comendador Roberth" dar conferencias de hipnotismo en el teatro Odeón. Según el artículo en cuestión, "el conde de Das aplaude" la resolución de la oficina de higiene, pues según su parecer es "muy peligrosa la presentación de fenómenos hipnóticos, reales o supuestos, ante un público compuesto muchas veces de personas exageradamente impresionables".

Lo que Sgaluppi buscó con su visita a La Prensa fue diferenciarse de modo tajante de otro individuo que por esos mismos días hacía ante los porteños cosas muy parecidas a las suyas. Roberth -de quien no poseemos información biográfica- debía comenzar por esos días con su espectáculo en el teatro Odeón. Según un diario local, su show constaba de "a) Penetración del pensamiento ageno; b) Roberthianide y psicología experimental; c) Matemática recreativa; d) Hipnotismo, catalepsia y espiritismo; e) Memoria e historia; f) El rompecabeza; g) Escenas humorísticas" ${ }^{29}$.

Unas pocas semanas después Sgaluppi tendría que enfrentarse a una medida represiva análoga, pero él contaba con herramientas bastante versátiles para hacer frente a la prohibición. Por lo pronto, el visitante se concentraba en los preparativos de la fundación de un instituto. Esa sociedad le permitiría remarcar aún más ante los ojos de los porteños cuál era su lugar de enunciación: él no era un vulgar hombre de teatro, y era capaz de combinar de modo fructífero lo más novedoso de la ciencia mental (la psicología) con un marco teórico que rehuyera de la obstinación del materialismo.

A mediados de diciembre, los órganos de prensa de la ciudad volvieron a ocuparse masivamente de Sgaluppi, pues el día 15 quedó inaugurado el Instituto Psicológico Argentino, cuya sede se encontraba en el segundo piso de la casa ubicada en Rivadavia 1777. El día de la inauguración tuvo lugar una conferencia especial, así como algunos experimentos. Los diarios más importantes ofrecieron largas reseñas sobre el acontecimiento. La Prensa advertía que a la velada habían asistido "algunas distinguidas familias y muchos caballeros de ilustración reconocida" ${ }^{30}$. Según la misma crónica, Sgaluppi hizo una intervención teórica, en la cual efectuó un singular sincretismo: bajo la divisa del "más allá" hizo desfilar los nombres de Newton, los oráculos, las esfinges, los fluidos etéreos o la corriente acásica de los judíos. A la hora de listar los conceptos con los que se sentía más próximo, se refirió al "limbo luminoso que rodea a cada persona" y definió a la enfermedad como la destrucción de la armonía. Esa enumeración heteróclita le servía para aclarar que en su trabajo no se trataba del hipnotismo "como algunos lo han dado a entender, algunos incrédulos e ignorantes". A los fines de dejar bien en claro que él no era uno de esos hipnotizadores teatrales que tanta animadversión provocaban entre los higienistas porteños, Sgaluppi reclamó silencio cada vez que el público intentaba aplaudir, señalando que "en el templo de la ciencia no se deben escuchar aplausos" ${ }^{\prime \prime}$. Tras la exposición doctrinal, vinieron las experiencias, realizadas sobre la mujer del anfitrión (sugestiones hipnóticas, rigidez cataléptica, transmisión del pensamiento a distancia, etc.).

La Nación informó asimismo que el Instituto haría las veces de una clínica médica tradicional: además de encargarse de la "enseñanza técnica y experimental de la psicología y de la psico-física moderna", se establecería un dispositivo para la asistencia de los enfermos presentados por los socios. Lo informado por La Nación era cierto. La apertura de una tal clínica figuraba en los Estatutos Generales redactados por los miembros del Instituto y distribuido por esos días (en forma de un folleto de 16 páginas) ${ }^{32}$. En ese documento quedaba expresamente indicado que el Instituto se fundaba por iniciativa del "Dr. D. Alberto Martínez de Das" y que su objeto era "la enseñanza teórica y experimental de la Psicología y de la Psico-física moderna". En todo momento se recalcaba que se trataba de una sociedad con fines científicos. Ese afán de cientificidad se comprueba sintomáticamente por aquello que quedaba expresamente prohibido, incluso desde los primeros artículos de la sección "Objeto de la sociedad": se prohibía toda discusión religiosa o política, y estaba por entero vedado todo experimento realizado con fines de pasatiempo o curiosidad. No podemos negar que existe una suerte de contraste 0 paradoja entre esas cautelas cientificistas y las faenas efectuadas por el fundador en las semanas previas, cuyas sesiones de hipnosis y telepatía tenían muchos parecidos con los elementos que ahora figuraban en el listado de las prohibiciones.

En esa fuente, así como en las crónicas periodísticas antes citadas, aparecía asimismo la composición del "Consejo Directivo". Había allí espiritistas militantes (Cosme Mariño y Felipe Senillosa). No obstante, figuraban también individuos que se habían ganado cierto prestigio en ámbitos científicos y profesionales. De un lado encontramos a Alejandro Sorondo (1856-1934), geógrafo y varias veces presidente del Instituto Geográfico Nacional -poco después sería la figura emblemática de la teosofía a nivel local-. Estaba además el ingeniero Rodolfo Moreno (1852-1929), que ocupaba nada menos que el cargo de presidente del Instituto Psicológico, y que desde hacía años se desempeñaba como docente de matemáticas en distintas universidades (Cutolo, 1968, 673). Debemos destacar asimismo al 
Vice-Presidente del centro, el filólogo de origen español Baldmar Dobranich (1853-1912), considerado hoy en día como uno de los principales precursores de la filología histórica en la Argentina (Lidgett, 2011). Encontramos también a profesionales del ámbito de la salud. De un lado, se desempeñaba como vocal Roberto Cárcamo (1868-1936), un doctor en Química y Farmacia de origen español, que a comienzos del siglo XX ocupó un lugar destacado en la historia de la farmacia del Río de la Plata. De otro lado, al menos dos médicos integraron el Instituto fundado por Das: Severino Pérez Redondo (Administrador) y José Popolizio (vocal) ${ }^{33}$.

No ha de sorprendernos la confluencia que en el Instituto se produjo entre defensores de credos esotéricos (como el espiritismo) e intelectuales o profesionales que podían respetar los hábitos y lenguajes de sus propias disciplinas científicas. En efecto, diversas investigaciones han documentado de modo convincente cuán atractivos podían resultar los espiritualismos cientificistas (karcecismo, teosofía, etc.) a los ojos de distintos actores del escenario cultural del cambio de siglo. Escritores, publicistas y científicos de ese período encontraron en aquellos espiritualismos un credo y un ideario merced al cual podían canalizar inquietudes e interrogantes que no hallaban cabida en las fronteras de la ciencia clásica (Quereilhac, 2010). Ahora bien, la presencia de médicos en aquella iniciativa auspiciada por el conde nos plantea algunos interrogantes. ¿Por qué motivo esos doctores se asociaron a un individuo que además de explotar teatralmente el hipnotismo y la telepatía, echaba mano en sus escritos a un vocabulario que parecía reñido con la medicina académica? A continuación veremos que otros colegas también siguieron esos pasos, estableciendo alianzas con Das incluso después de que éste fuera reprendido severamente por las autoridades sanitarias, quienes muy pronto pusieron en entredicho que el visitante poseyera título médico ${ }^{34}$.

\section{DE LA REPRESIÓN HIGIÉNICA A LA ALIANZA CON UN MÉDICO}

Tanto por las acciones de Sgaluppi de las semanas previas, como por el contenido de los Estatutos, es evidente que el conde y sus correligionarios entendían cuán necesario era convencer a las autoridades sanitarias sobre sus buenas intenciones. Reiterando su autodefinición como médico, Sgaluppi parecía dirigir a los higienistas un doble mensaje articulado. Primero, mediante la advertencia de que el hipnotismo no sería el centro de las actividades del Instituto-advertencia explicitada durante la conferencia de inauguración, y reforzada en los Estatutos, en cuyas páginas los términos de hipnosis o magnetismo apenas asoman-, buscaba de alguna forma tranquilizar los temores de las autoridades higiénicas. Segundo, y de algún modo a cambio de su buena conducta, se atrevía a solicitar a esas mismas autoridades un poco de terreno de acción. En efecto, según las crónicas, al comienzo de su conferencia afirmó que los resultados del Instituto "serían positivos si se le abriesen tres puertas que estaban cerradas para ellos: el hospital, el manicomio y las cárceles" 35 .

Si medimos la iniciativa a la luz de las reacciones que suscitó, podemos concluir que sus responsables actuaron con poca cautela. Si bien Das podía prometer no infringir las normas vigentes, a los ojos de los porteños resultaba más que evidente que entre el Instituto recién inaugurado y las sociedades espiritistas existían lazos muy estrechos. Las noticias que unos días más tarde publicarían los miembros de Constancia dejaban al descubierto ese parentesco, inaceptable para las autoridades sanitarias, sobre todo desde el momento que el Instituto prometía habilitar una clínica. En efecto, en el primer número de 1893 de la revista espiritista encontramos una reseña entusiasta sobre la inauguración del Instituto, en la cual se señalaba la comunidad de intereses: "el Instituto Psicológico Argentino será una nueva fuente de estudio, que por otros senderos quizás, nos conducirá al mismo resultado, por lo menos en lo que respecta a la demostración positiva de la existencia del alma, base fundamental de la doctrina espiritista" ${ }^{36}$.

Las autoridades no tardaron en reaccionar a la iniciativa de Sgaluppi. Una vez más, conviene recordar aquí algunos pormenores contextuales. Es probable que los responsables del control sanitario hayan querido actuar de inmediato, antes de que la ciudad viera emerger un nuevo consultorio hipnoterápico dirigido por un profano. Desde fines de 1889 el español Alberto Díaz de la Quintana (que carecía de título de doctor, y que se negaba a revalidar su título en el país) dirigía en la ciudad un "Gabinete hipno-terápico", que había logrado un gran éxito entre los porteños. A pesar de repetidas multas y advertencias, las autoridades sanitarias jamás lograron la clausura de ese establecimiento (que cerraría sus puertas en 1893, no debido a la represión sino al regreso a España de su dueño) (Vallejo, 2015). Más aún, en los mismos días en que Sgaluppi realizaba sus primeras demostraciones en Buenos Aires, Díaz de la Quintana había vuelto a dar dolores de cabeza a las autoridades, pues había conseguido una patente de invención por un instrumento terapéutico vibratorio diseñado por él. Ese episodio había desencadenado una disputa entre oficinas del gobierno, pues el Departamento de Higiene lamentó que la Oficina de Patentes no lo hubiera consultado antes de dar el visto bueno a la innovación presentada por Díaz de la Quintana. En síntesis, es posible que los miembros de la oficina de higiene tuvieran presente el caso del director del "Gabinete hipno-terápico" cuando decidieron reaccionar sin demora a la reciente fundación del Instituto Psicológico Argentino. 
El 17 de diciembre algunos diarios informaron que el jefe del Departamento Nacional de Higiene, José María Ramos Mejía (1849-1914), había solicitado al jefe de policía de la ciudad que prohibiera las conferencias que Martínez de Das tenía pensando realizar próximamente ${ }^{37}$. Un órgano de prensa en particular, Tribuna, dedicó una larga columna a respaldar esa decisión ${ }^{38}$. Tenemos algunas evidencias sobre lo sucedido inmediatamente después de esa medida represiva. Sabemos, para empezar, que Sgaluppi y sus colegas del Instituto intentaron resistir el ataque. Lo hicieron sobre todo recurriendo a los órganos de prensa. Al día siguiente, El Correo Español reprodujo una carta enviada al diario por el secretario general del Instituto, Alexis Curutchet, cuyo fin era realizar algunas precisiones $^{39}$. De un lado, se aclaraba que Martínez de Das no actuaba a título personal, sino en calidad de profesor nombrado por el Instituto. De otro lado, que los "cursos" ofrecidos por la institución no eran públicos, sino que estaban reservados para los individuos que se atuvieran al reglamento del Instituto. Por último, que el consejo directivo era el encargado de determinar quiénes, "por su condiciones de saber", debían ser admitidos para presenciar los experimentos.

El desenlace del altercado resulta complejo. Cuatro días más tarde, y sin ocultar su satisfacción al respecto, el diario de la comunidad española informaba que el jefe de policía había hecho caso omiso del pedido del Departamento de Higiene. Según la crónica, hacía algunos días aquel jefe había respondido a las autoridades higiénicas que no estaba dentro de sus facultades el prohibir conferencias como las de Martínez de Das ${ }^{40}$. Ese mismo día, el vespertino El Diario desmentía la información difundida por El Correo Español, aclarando que la nota enviada por Ramos Mejía a la policía "será tomada en consideración como es debido" ${ }^{41}$. En ese mismo artículo por primera vez se ponían en duda los títulos alegados por Sgaluppi. En efecto, se agregaba que el director de la oficina de higiene entendía que Das no era médico, y que por ese motivo no estaba habilitado para dictar sus conferencias.

Quizá El Diario estaba en lo cierto, y la policía no cuestionó el pedido de la oficina de higiene. Lo seguro es que, haya intervenido o no la fuerza policial, Das continuó ofreciendo conferencias, y el Instituto siguió adelante con sus actividades. Era normal que así fueran las cosas. Los historiadores que se han ocupado de la persecución del curanderismo en Buenos Aires a fines del siglo XIX, han documentado ya las dificultades legales y administrativas que existían para reprimir eficazmente el accionar de los competidores de los médicos (Macagno, 2008) ${ }^{42}$. La práctica habitual era que los perseguidos fueran sancionados con una multa; luego de pagarla, volvían a abrir sus consultorios u ofrecer sus servicios. Sin ir más lejos, ya hemos indicado que en ese mismo momento Alberto Díaz de la Quintana jamás tuvo que cerrar sus "Gabinete hipno-terápico" a pesar de las reiteradas multas y de las acusaciones de ejercicio ilegal de la medicina. Lo mismo podría señalarse respecto de la aplicación de una segunda normativa que seguramente se quiso hacer valer sobre Sgaluppi, la referida a explotación teatral del hipnotismo. También sabemos que su puesta en vigor fue dificultosa ${ }^{43}$

Al menos hasta finales de enero de 1893, Sgaluppi prosiguió sus tareas. Para el 3 de enero se anunció su conferencia "La conciencia íntima. El bien y el mal", que iría acompañada de "demostraciones al efecto", y a la cual podrían asistir solamente los socios ${ }^{44}$. Una semana más tarde tuvo lugar "la conferencia habitual del doctor" Das, esta vez sobre "La creencia magnética, el éter inteligente y la fuerza inter-etérica" ${ }^{45}$. Según el diario más exitoso de la ciudad, el Instituto contaba cada día con "mayor número de adeptos".

Ha llegado hasta nosotros el contenido de la primera de esas dos conferencias ${ }^{46}$. En ella se vuelve absolutamente transparente algo que ya se adivinaba en el escrito "Salve", aparecido unas semanas antes: la prédica de Sgaluppi poco tenía que ver con el lenguaje o los objetos de saber de la medicina, y mucho en cambio con el vocabulario y los enunciados del espiritualismo y las ciencias ocultas. Si bien en este texto Sgaluppi volvía a criticar el uso del hipnotismo con fines de esparcimiento, no había posibilidades de construir un lenguaje común con la medicina. Los objetivos y premisas del discurso de Sgaluppi eran por el contrario muy afines al ideario espiritista, contrario al materialismo, y anclado en una prédica moral sobre la autosuperación.

Hacia fines de enero de 1893 el nombre de Sgaluppi desaparece de los diarios de la ciudad. Sólo la prensa espiritista seguirá ocupándose de sus faenas. Por ejemplo, en el número del 29 de enero de Constancia, Ovidio Rebaudi afirmaba que el Instituto seguía funcionando a pesar de los intentos de las autoridades higiénicas por clausurarlo, y a pesar del apoyo que el sector católico había dado a esa medida represiva (Rebaudi, 1893).

El Departamento de Higiene finalmente logró su cometido, y el Instituto tuvo que cerrar sus puertas en febrero de 1893. Pero su director no se dio por vencido. Junto con fundar, en junio de 1893 , la Sociedad Luz, primera rama local de la Escuela Teosófica -punto al cual volveremos en unos instantes-, Sgaluppi prosiguió sus demostraciones de hipnotismo y sus conferencias. Quizá como resultado de las persecuciones sufridas unos meses antes, realizó estas últimas tareas en colaboración de un médico local, Osvaldo García Piñeiro ${ }^{47}$. Una vez más, estamos ante una táctica muy utilizada por quienes eran acusados de ejercicio ilegal de la medicina. Vale aquí nuevamente la comparación 
con la conducta del hipnotizador Díaz de la Quintana. Luego de haber pagado reiteradas veces la multa por ejercicio ilegítimo del arte de curar, este último colocó como "Médico Director" de su gabinete al "Dr. G. De Rossi" 48 . Sea como fuere, sabemos que a partir de julio de 1893 Sgaluppi y García Piñero actuaron juntos en un par de ocasiones. A principios de julio de ese año, en el domicilio de Sgaluppi (Callao 341) y ante una asistencia de casi 80 personas, ambos hipnotizadores hicieron sus demostraciones ${ }^{49}$. En primer instancia, García Piñero exhibió fenómenos hipnóticos con la colaboración de tres pacientes mujeres curadas por él hacía poco. A continuación tomó la palabra Sgaluppi, y se refirió al poder de los fluidos, tras lo cual hipnotizó a su esposa, quien en ese estado mostró capacidades telepáticas. Dos meses más tarde, y en el mismo lugar, tuvo lugar una segunda conferencia, en la cual en realidad sólo Sgaluppi disertó (esta vez sobre teosofía y ciencias de oriente) $)^{50}$. En el cierre de la crónica, se informaba que la asociación entre Sgaluppi y García Piñero se había fortalecido y materializado en la apertura de una clínica terapéutica: "Después de las experiencias, pasaron los concurrentes a visitar una instalación de baños eléctricos, y de nuevo sistema, y de cuya eficacia tanto el Dr. Das como el Dr. García Piñeiro esperan muy buenos resultados".

\section{A MODO DE CIERRE}

Todo lo referido a la fundación de la rama $L u z$, primera filial argentina de la teosofía, ya ha sido analizado por Soledad Quereilhac (2010). A los fines de fundar la Sociedad, Sgaluppi se dirigió al Coronel Olcott en la India, máxima autoridad del movimiento teosófico, utilizando el nombre "Dr. A. Martinez". Obtuvo de la India el diploma y la Carta Constitutiva. Después de ello, cometió la torpeza de comunicarse por carta con los teósofos españoles, a los fines de solicitar apoyo en su nueva empresa y olvido de las faltas pasadas. Enterados los españoles de que se trataba del mismo sujeto que tanto escándalo había producido entre sus filas un año antes, advirtieron a Olcott, quien decretó la re-expulsión inmediata de Sgaluppi ${ }^{51}$. Allí se inicia el largo periplo latinoamericano de Sgaluppi, que mereció años después un colorido texto de José Carlos Mariátegui (1915).

En este cierre quisiéramos hacer un balance de las evidencias desplegadas a lo largo del artículo. Ante todo debemos retomar la pregunta sobre el motivo por el cual algunos médicos de Buenos Aires secundaron al conde en sus emprendimientos, incluso después de que tomara estado público que aquel carecía de título profesional. A nuestro entender, es legítimo plantear para el caso de Buenos Aires la hipótesis esgrimida por Andrea Graus en su estudio sobre el hipnotismo en España (Graus, 2017). En ambos territorios, los hipnotizadores no diplomados mostraron una gran pericia en el manejo práctico de la hipnosis, al tiempo que los médicos estaban poco familiarizados con esa herramienta. En efecto, estos últimos no hallaban en las universidades $u$ hospitales un adiestramiento en esa materia, y estaban de alguna forma obligados a buscar en escenarios alternativos una introducción a ese arte. Más aún, para el caso de la capital argentina, los profanos o no diplomados les llevaban la delantera a los médicos no sólo en lo que se refiere al manejo práctico de la hipnosis, sino también en lo que hace a la elaboración teórica respecto de esos fenómenos (Vallejo, 2014). Dicho en otros términos, a la hora de examinar la historia del hipnotismo en lugares como Buenos Aires, y sobre todo al momento de indagar las relaciones o tensiones mantenidas entre los médicos y sus competidores profanos, es menester atender no tanto a las estrategias desplegadas por los primeros a los fines de conseguir un monopolio en el ejercicio y la explicación de esa herramienta curativa, sino más bien a los mecanismos (de vigilancia, pero también de emulación) merced a los cuales los doctores sacaron provecho de las pericias y capacidades de sus contrincantes.

Creemos que el análisis de las tareas de Sgaluppi en Buenos Aires pone de relieve esa dimensión hasta ahora poco atendida por los historiadores de la medicina local. Los episodios recuperados aquí iluminan en igual medida otros aspectos de la cultura científica de fin de siglo. Primero, para poner de relieve una vez más la naturalidad con que idearios esotéricos podían seducir al público ilustrado de la ciudad capital. Médicos, ingenieros y hombres de letras no solamente asistieron con placer a demostraciones de telepatía, sino que participaron activamente en un emprendimiento asociativo encargado de divulgar conocimientos sobre esas materias. Segundo, muestran la relativa libertad de acción que este tipo de individuos tuvieron en el mercado de la salud durante esas décadas. Estos hipnotizadores, ilusionistas, curanderos o magnetizadores fueron personajes habituales en el Buenos Aires del cambio de siglo, y en todos los casos es fácil reconstruir cuán ineficaces fueron los intentos realizados por las autoridades sanitarias para prohibir su accionar.

Existe, para concluir, un último elemento que quisiéramos resaltar con especial dedicación. En el comienzo del artículo colocamos al Instituto fundado por Sgaluppi como una suerte de antecedente olvidado de las futuras sociedades de psicología en Argentina. Al emitir esa afirmación quedamos expuestos a una objeción muy grave: lo "psicológico" de aquel Instituto parece no tener nada que ver con la psicología, tal y como ella se constituiría por esos años en los ámbitos académicos. En efecto, es menester recordar que durante esas décadas lo "psíquico" o lo "psicológico" eran muchas veces términos de las ciencias de lo paranormal, y que la psicología científica nació con otros 
objetivos. Ahora bien, diversas investigaciones recientes han mostrado que esa divisoria de aguas nunca fue segura (Lachapelle, 2011; Plas, 2012). El hecho de que la psicología científica del siglo XX haya optado, al comienzo de su recorrido, por descartar toda la fenomenología tildada de paranormal -categoría en que se colocaron los hechos afines al hipnotismo-, no habilita para desconocer u olvidar que durante cierto tiempo ambos universos marcharon de la mano, o que los foros de lo paranormal jugaron un papel clave en la construcción de objetos de saber o en la difusión de autores y teorías que fueron esenciales para el desenvolvimiento de la psicología. Sin ir más lejos, eso mismo podría ser argüido para el caso que nos ocupa. El espiritismo (con sus revistas, sus reuniones, sus actividades de divulgación), y en menor medida el Instituto Psicológico, dieron visibilidad a hechos y autores que luego serían reapropiados por los primeros artífices de la psicología local. Más aún, la referencia a José Ingenieros puede resultar aquí muy ilustrativa. Quien a comienzos de siglo XX se transformaría en la figura más importante de la psicología científica en Buenos Aires, participó previamente de las actividades de la

\section{NOTAS}

1. Entre los numerosos trabajos referidos a la historia de la psicología en Argentina, el temprano escrito de René Gotthelf es el único que menciona superficialmente la fundación en 1892 del Instituto (Gotthelf, 1969).

2. Fue el caso de los miembros de las sociedades espiritistas de Buenos Aires, quienes se transformarían en los principales defensores de Sgaluppi. Para los kardecianos porteños, el nombre de nuestro personaje era muy conocido (incluso desde el momento mismo en que se iniciaron sus demostraciones en España, a mediados de 1887), sobre todo debido a las crónicas positivas que se publicaban sobre sus trabajos en Barcelona o Madrid; véase, a modo de ejemplo, "Hipnotismo", La Fraternidad. Revista quincenal, Año VI, N. 20, 19 de Junio de 1887, p. 1091; Ovidio Rebaudi, "Apuntes sobre el fluido magnético, base de los fenómenos que nos presentan el magnetismo y el espiritismo", Constancia. Revista quincenal, espiritista bonaerense, Año XIII, N 208, 15 de agosto de 1890, pp. 227-232.

3. El Imparcial, 9 de enero de 1888, p. 3.

4. La Monarquía, 14 de enero de 1888, p. 1; El Siglo Futuro, 17 de enero de 1888 , p. 2 . Si bien las crónicas se refieren a Isabel como la "Reina Regente", cabe aclarar que no poseía esa investidura desde 1868. María Cristina fue quien ejerció la regencia entre 1885 hasta 1902

5. La Dinastía, 30 de enero de 1888, p. 2.

6. Según informó La Iberia, el primer número habría aparecido el 5 de febrero de 1888 (La Iberia, 11 de febrero de 1888, p. teosofía porteña. No sólo publicó en una de sus revistas (Philadelphia) algunos fragmentos de lo que luego sería su libro Los accidentes histéricos y las sugestiones terapéuticas, sino que a través de esos órganos de difusión tomó conocimiento de autores y conceptos ligados a la problemática de lo mental (Quereilhac, 2010). No quisiéramos incurrir en argumentos contrafácticos -y preguntar qué hubiese sido de Ingenieros y sus ideas psicológicas sin su temprana adhesión a la teosofía-, pero es evidente que él, al igual que otros médicos e intelectuales, hallaron en las iniciativas del cientificismo esotérico una valiosa fuente de saberes y evidencias referidos al automatismo nervioso, las fuerzas psíquicas enigmáticas o los límites de la voluntad. Es también seguro que sin la visita de Sgaluppi esa teosofía hubiera tomado más tiempo en llegar, y que los estudios sobre el hipnotismo hubieran tenido menos visibilidad en la ciudad. Es por ello que la reconstrucción de las acciones de sujetos como Sgaluppi puede ayudar para tener un conocimiento más cabal de esas zonas donde la historia de la psicología, la historia de la medicina y del esoterismo convergieron de modo provechoso.

2). En esa misma columna se reprodujo el índice del primer número, el cual contenía un escrito de Das titulado "Hipnotismo y zoomagnetismo" -trabajo que unas semanas más tarde sería reproducido en una revista de divulgación (Das, 1888). En la revista de hipnosis más importante de aquellos años, la Revue de l'hypnotisme publicada en Francia, apareció una pequeña noticia sobre La Hipnoterapia. Junto con comunicar que habían recibido "los primeros números", los redactores se permitían un llamado de atención a su director, el "doctor Don Alberto de Diaz (sic)": le advertían que en aquellas páginas se concedía título de "doctor" o "profesor" a colaboradores que probablemente carecían de esas credenciales, y eran seguramente meros magnetizadores profanos ("Nouvelles", Revue de l'hypnotisme expérimental et thérapeutique, Año II, 1888, p. 288).

7. La Época, 5 de febrero de 1888, p. 2.

8. La Época, 7 de febrero de 1888. Véase también Aycart (1888) y Graus (2014).

9. Véase, entre otros, La Época, 26 de diciembre de 1890, p. 4.

10. "Crónicas madrileñas", La Ilustración Hispano-Americana, Año XII, № 532, 11 de enero de 1891, p. 18

11. “Advertencia”, Estudios Teosóficos, Serie 2, №14, p. 341.

12. De forma reciente han aparecido algunos trabajos en los que quedan referidas las exhibiciones de hipnotismo realizadas por Das en Madrid (González de Pablo, 2016; Graus, 2017). Especial importancia para nuestro enfoque posee el artículo de Andrea Graus, pues allí se postula y documenta 
la hipótesis que defendemos: las labores de Das, así como la de otros hipnotizadores no diplomados de aquella época, no debe ser menospreciada, pues ellos oficiaron muchas veces de verdaderos maestros de los médicos. Estos últimos aprendían la técnica hipnótica observando las exhibiciones de aquellos 'profanos' y competidores, e incluso asistían a las lecciones que impartían (Graus, 2014).

13. "Crónica general”, La ilustración española y americana, Año XXXII, № VI, 15 de febrero de 1888, p. 106. Una idea similar es planteada en "La Sociedad Española de Higiene y el hipnotismo", La Unión católica, 21 de febrero de 1888, p. 2. El fuerte protagonismo de Das en la popularización del hipnotismo fue señalado incluso en el libro El hipnotismo en moda publicado en 1889 por Juan José Franco (Franco, 1889: 46-49).

14. La Iberia, 19 de Noviembre de 1888, p. 2. Véase también "Sesión de la Sociedad Española de Higiene. Sobre los hipnotismos", El Imparcial, 15 de febrero de 1888, p. 3; "La Sociedad Española de Higiene y el hipnotismo", La Unión Católica, 21 de febrero de 1888, p.2; Alvaro López Nuñez, "El hipnotista andante", La Ilustración Católica, Época 6, Año XVI, Tomo XIV, 15 de marzo de 1891, pp. 68-70.

15. Eugenio Fernández Hidalgo, "El Diablo en Madrid. Las representaciones hipnóticas", La Unión Católica, 22 de febrero de 1888, p. 1.

16. “El Dr. A. de Martínez", La Nación, 29 de octubre de 1892, p. 3.

17. "Psiquiatría - Conferencia experimental", La Nación, 31 de octubre de 1892, p. 3.

18. "Hipnología”, El Correo Español, 1 de noviembre de 1892, p. 2.

19. "Conferencia psiquiátrica", El Diario, 1 de noviembre de 1892 , p. 2.

20. "Conferencia sobre psiquiatría", La Prensa, 1 de noviembre de 1892, p. 2.

21. "La conferencia del Dr. Martínez de Das", La Nación, 1 de noviembre de 1892, p. 1. Esa misma crítica figura "Hipnotismo", Tribuna, 1 de noviembre de 1892, p. 2.

22. De todas esas demostraciones anteriores, algunas ya han sido reconstruidas con cierto detalle: las sesiones dadas en agosto de 1888 por el médium Henry Slade, los espectáculos de hipnosis desarrollados por Guyot de Thorcey en un teatro de la ciudad en los meses finales de 1889 o los shows con similar contenido dados por el prestidigitador Enireb en octubre de 1891 (véase Mariño, 1931, 143-156; Fernández, 1996, 322-337).

23. La resolución fue publicada en los Anales del Departamento Nacional de Higiene, Año 1, № 5, 303-304. Su sanción, efectuada en el mismo momento en que normativas similares aparecían en otros países, respondió seguramente al deseo de los médicos de ejercer un control o monopolio de una herramienta curativa muy explotada por los sanadores nodiplomados.

24. Fue el caso del español Alberto Díaz de la Quintana, que en Buenos Aires ofreció sesiones públicas de hipnotismo con el auxilio de una paciente, Carolina del Viso (Vallejo, 2015). En los últimos años han visto la luz valiosas investigaciones referidas a los cruces entre lo científico y lo espectacular. Para ese terreno de estudios en América Latina, nos remitimos al volumen Ciencia y espectáculo publicado hace poco (Correa, Kottow y Vetö, 2016).

25. Uno de los síntomas inmediatos de su popularidad fue que muy pronto su nombre apareció incluso en una publicación satírica de Buenos Aires; véase "A la minuta", La Caricatura. Semanario ilustrado, político y literario, Año 2, № 49, 7 de noviembre de 1892, p. 3.

26. Dr. A. M. de Das, "Salve", Constancia, Año XV, № 281, 13 de noviembre de 1892, p. 291.

27. Lucio Meléndez (1844-1901) fue la figura más destacada del alienismo argentino de la segunda mitad del siglo XIX. Durante casi 20 años (1875-1892) dirigió el manicomio de hombres (Hospicio de las Mercedes), y fue el titular de la primera cátedra de Enfermedades Nerviosas (entre 1886 y 1893) de la Facultad de Medicina de la Universidad de Buenos Aires (Vezzetti, 1983). Wilfrido Rodríguez de la Torre (1859?-1890), por su parte, fue un médico proveniente de la ciudad de Córdoba, autor de numerosos trabajos sobre neurología. Hacia abril de 1890, unos meses después de editado su tratado sobre el espiritismo, falleció a temprana edad.

28. “Ciencia psicológica”, La Prensa, 20 de noviembre de 1892, p. 2.

29. "El Profesor Roberth", El Correo Español, 18 de noviembre de 1892 , p. 2.

30. "Instituto Psicológico Argentino. La conferencia de anoche", La Prensa, 16 de diciembre de 1892, p. 2.

31. "Instituto Psicológico. Sesión inaugural”, La Nación, 17 de diciembre de 1892, p. 1.

32. Estatutos Generales. Reglamento interior. Instituto Psicológico Argentino. Buenos Aires: Roma.

33. Severino Pérez Redondo revalidó su título en el país en 1889 ("Nómina de los médicos que han revalidado sus títulos en esta Facultad desde el 1 de enero de 1888 hasta el 1 de enero de 1893", Anales del Departamento Nacional de Higiene, Año III, 7, Septiembre de 1893, p. 302). José Popolizio se doctoró en Buenos Aires en 1887 con una tesis sobre la disentería.

34. "Conferencias prohibidas", El Diario, 22 de diciembre de 1892, p. 2 
35. "Instituto Psicológico. Sesión inaugural", La Nación, 17 de diciembre de 1892, p. 1.

36. "El Instituto Psicológico Argentino", Constancia, Año XVI, № 288, 1 de enero de 1893, p. 346.

37. "Las conferencias psicológicas", La Prensa, 17 de diciembre de 1892, p. 3.

38. "El consejo de higiene y la psiquiatría", Tribuna, 17 de diciembre de 1892, p. 2.

39. "Cómo se pide", El Correo Español, 18 de diciembre de 1892, p. 2

40. "Petición negada", El Correo Español, 22 de diciembre de 1892, p. 2.

41. "Conferencias prohibidas", El Diario, 22 de diciembre de 1892, p. 2.

42. De todas esas dificultades (referidas, por ejemplo, a la ineficacia de la medicina diplomada para sanar un gran cúmulo de enfermedades, o a la carencia de médicos profesionales en pequeños pueblos o zonas rurales), conviene remarcar la siguiente: recién en 1921, gracias a la reforma del Código Penal, el curanderismo se convirtió en un delito. Hasta ese entonces era considerado una mera contravención, que podía ser reparada mediante una multa en dinero.

43. La primera vez que se quiso poner en práctica la ordenanza sobre hipnotismo teatral (sancionada en diciembre de 1890), no se logró nada. En efecto, en mayo de 1891 un célebre ilusionista, Aquiles Regis, desembarcó en Buenos Aires. Advertido de la existencia de aquella ordenanza, dirigió al Departamento de Higiene una nota pidiendo permiso para realizar su espectáculo de adivinación del pensamiento, alegando que no tenía nada que ver con la hipnosis. Su pedido fue negado ("Policía médica", Anales del Departamento nacional de Higiene, Año I, № 5, 293-295). A pesar de ello, brindó sus shows en los salones del Operai Italiani,

\section{BIBLIOGRAFÍA}

Aycart, Luis (1888), “Dos sesiones distintas y un solo espectáculo verdadero", Revista de Sanidad Militar, 2 (16), pp. 52-60.

Correa, María José; Kottow, Andrea y Vetö, Silvana (2016) Ciencia y espectáculo. Circulación de saberes científicos en América Latina, siglos XIX Y XX, Santiago de Chile, Ocholibros.

Cutolo, Vicente (1968), Nuevo diccionario biográfico argentino (1750-1930), Buenos Aires, Elche.

Das, Alberto de (1888), "Hipnotismo y zoomagnetismo", Revista popular de conocimientos útiles, XXX (386), pp. 85-86.

Diéguez Gómez, Antonio (2003), “Hipnotismo y medicina mental en la España del siglo XIX". En: Montiel, Luis y González de Pablo, Ángel (eds.), En ningún lugar en parte alguna: Es- que fueron reseñados por los espiritistas (Constancia, Año XIV, N²28, p. 179).

44. "Instituto Psicológico Argentino", El Correo Español, 1 de enero de 1893, p. 2; "Instituto Psicológico Argentino", La Prensa, 3 de enero de 1893, p. 3.

45. "Instituto Psicológico Argentino", La Prensa, 10 de enero de 1893, p. 2.

46. El texto íntegro de la conferencia fue poco después publicado en la revista espiritista; véase Dr. Martínez de Das, "La conciencia íntima", Constancia, Año XVI, № 290, 15 de enero de 1893, pp. 21-23.

47. Es poca la información que hemos podido recoger sobre este doctor. Sabemos que obtuvo su título en 1886 mediante una tesis titulada Porvenir nosográfico de la ciudad de Buenos Aires: embriaguez. Se dedicó a la aplicación del hipnotismo curativo durante muchos años, experiencia recogida en 1902 en un folleto titulado Curaciones por el hipnotismo (García Piñeiro, 1902).

48. La figura del "Médico Director" aparece en las publicidades del gabinete recién a fines de 1892; véase, a modo de ejemplo, La Nación, 19 de octubre de 1892, p. 3. Es casi seguro que a esa artimaña de Díaz de la Quintana se refiere un artículo publicado en El Diario en diciembre de ese año, en el cual se protesta justamente contra ese tipo de asociaciones; "Curanderismo. Ejercicio ilegal de la medicina", El Diario, 28 de diciembre de 1892, p. 2.

49. Véase una crónica detallada en "Sección científica", Constancia, Año XVI, N³17, p. 257.

50. "Noticias generales", Constancia, Año XVI, № 325, pp. 321322.

51. La carta figura en "Reexpulsión de D. Alberto de Das (Conde de Das) de la Sociedad Teosófica", Sophia. Revista Teosófica, Madrid, octubre 1893, pp. 242-244.

tudios sobre la historia del magnetismo animal y del hipnotismo, Madrid, Frenia, pp. 197-228.

Fernández, Mauro (1996), Historia de la magia y el ilusionismo en la Argentina, Buenos Aires, s./d.

Franco, Juan José (1889), El hipnotismo en moda. Estudio histórico y crítico, Lugo, Juan María Bravos.

García Piñeiro, Osvaldo (1902), Curaciones por el hipnotismo, Buenos Aires, Imp. y casa editora de A. Etchepareborda.

González de Pablo, Ángel (2003), “El hipnotismo en la España del primer tercio del siglo XX". En: Montiel, Luis y González de Pablo, Ángel (eds.), En ningún lugar en parte alguna: Estudios sobre la historia del magnetismo animal y del hipnotismo, Madrid, Frenia, pp. 229-297. 
González de Pablo (2016), “Consolidar, colonizar, excluir: estrategias de legitimación de la hipnosis médica". En: Mülberger, Annette (ed.), Los límites de la ciencia. Espiritismo, hipnotismo y el estudio de los fenómenos paranormales (1850-1930), Madrid, CSIC, pp. 161-192.

Gotthelf, René (1969), "Historia de la psicología en la Argentina", Anuario de Historia del Pensamiento argentino, V, pp. 63-132.

Graus, Andrea (2014), "Hypnosis in Spain (1888-1905): From spectacle to medical treatment of mediumship", Studies in History and Philosophy of Bilogical and Biomedical Sciences, 48, pp. 85-93.

Graus, Andrea (2017), "Hypnosis lessons by stage magnetizers: Medical and lay hypnotists in Spain", Notes and Records: The Royal Society Journal of the history of science, 71 (1), pp. 141-156.

Lachapelle, Sofie (2011), Investigating the Supernatural. From Spiritism and Occultism to Psychical Research and Metapsychics in France, 1853-1931, Baltimore, John Hopkins University Press.

Lidgett, Esteban (2011), “Gramática escolar y políticas lingüísticas en la Argentina: un análisis de la Gramática de la lengua castellana (1893) de Baldmar Dobranich y Ricardo Monner Sans", Revista argentina de historiografía lingüística, 3 (2), pp. 109-132.

Macagno, Mauricio (2008), “Construyendo un delincuente: Ramos Mejía y el ejercicio ilegal de la medicina (sobre motivos e influencias)", Anales. Revista de la Facultad de Ciencias Jurídicas y Sociales de la Universidad Nacional de La Plata, 5, pp. 1-38.

Mariátegui, José Carlos (1915), “Un aventurero de folletín”. En: Mariátegui, José Carlos, Escritos Juveniles, 2: La edad de piedra, Lima, Amauta, pp. 67-72.

Mariño, Cosme (1931), El espiritismo en Argentina, Buenos Aires, Constancia.
Ostrovsky, Ana (2008), "La sociedad de psicología en Argentina (1908-1913): treinta y nueve hombres y una mujer", Revista de Historia de la Psicología, 29 (2), pp. 55-67.

Plas, Régine (2012), "Psychology and psychical research in France around the end of the $19^{\text {th }}$ century", History of the Human Sciences, 25 (2), pp. 91-107.

Quereilhac, Soledad (2010), La imaginación cientifica: ciencias ocultas y literatura fantástica en el Buenos Aires de entresiglos (1875-1910), Tesis doctoral, Universidad de Buenos Aires.

Rebaudi, Ovidio (1893), “El Instituto Psicológico Argentino y el Dr. Gamaliel”, Constancia, 14 (292), pp. 34-35.

Ríos, Julio y Talak, Ana María (1999), “La articulación entre el saber académico y diversas prácticas de la psicología, en la Sociedad de Psicología de Buenos Aires entre 1908 y 1913", Anuario de Investigaciones, 6, 382-391.

Rodríguez de la Torre, Wilfrido (1889), Espiritismo y locura. Sus relaciones recíprocas, Buenos Aires, Lajouane.

Sancha y Hervás, C. M. (1888), Carta pastoral del Excmo. é Ilmo Sr. Obispo de Madrid-Alcalá sobre el hipnotismo, Madrid, Tipografía de los Huérfanos.

Vallejo, Mauro (2014), "Buenos Aires mesmérica. Hipnosis y magnetismo en la cultura y la ciencia de la capital argentina (1870-1900)", Iberoamericana, 14 (56), pp. 7-26.

Vallejo, Mauro (2015), “Alberto Díaz de la Quintana y las tensiones del campo médico en Buenos Aires (1889-1892). Hipnosis, curanderismo y médicos extranjeros en la Argentina finisecular", Culturas Psi/Psy Cultures, 4, pp. 53-84.

Vezzetti, Hugo (1983), La Locura en la Argentina, Buenos Aires, Editorial Folios. 